\title{
Fractal analysis for the ULF data during the 1993 Guam earthquake to study prefracture criticality
}

\author{
Y. Ida and M. Hayakawa \\ Department of Electronic Engineering, The University of Electro-Communications, 1-5-1 Chofugaoka, Chofu, Tokyo, \\ 182-8585, Japan
}

Received: 16 January 2006 - Revised: 13 June 2006 - Accepted: 23 June 2006 - Published: 11 August 2006

\begin{abstract}
An extremely large earthquake (with magnitude of 8.2) happened on 8 August 1993 near the Guam island, and ultra-low-frequency (ULF) (frequency less than $1 \mathrm{~Hz}$ ) electromagnetic fields were measured by 3 -axis induction magnetometers at an observing station (with the epicentral distance of $65 \mathrm{~km}$ ) with sampling frequency of $1 \mathrm{~Hz}$. In order to study electromagnetic signature of prefracture criticality, we have undertaken the fractal (mono-fractal) analysis by means of the Higuchi's method for the ULF data during the 1993 Guam earthquake. Then, it is found that the fractal dimension exhibits five maxima 99, 75, 52, 21, and 9-4 days before the earthquake main shock, which suggests the ULF electromagnetic signature of nonlinear evolution (in the sense of self-organized criticality) taking place in the lithosphere just before the 1993 large Guam earthquake. That is, there take place step-like changes in the lithosphere during the longterm of the order of several months before the main shock.
\end{abstract}

\section{Introduction}

There have been recently a lot of reports on the presence of seismogenic emissions in a wide frequency range (Hayakawa and Fujinawa, 1994; Hayakawa, 1999; Hayakawa and Molchanov, 2002). Especially, ULF (ultra-low-frequency, frequency less than $1 \mathrm{~Hz}$ or so) emission is considered to be the most promising candidate for short-term earthquake prediction. While, higher frequency (VLF up to VHF) emissions are known to suffer from extremely large attenuation in the propagation in the Earth's crust, and then we have to suppose the secondary generation of those higher frequency emissions near the Earth's surface due to some unknown mechanism. On the other hand, the ULF emissions (and acoustic emissions) can be considered to directly reflect the informa-

Correspondence to: M. Hayakawa

(hayakawa@whistler.ee.uec.ac.jp) tion on microfracturing in the lithosphere (Hayakawa, 2001; Hayakawa and Hattori, 2004). ULF emissions are produced by microcracks (Molchanov and Hayakawa, 1995, 1998), which can be considered as the so-called precursors of general fracture of an earthquake.

Our main goal of this paper is to monitor the microfractures which are known to occur in final breakup in the focal zone of an earthquake by recording the ULF emissions. The presence of precursory signature of earthquakes is clearly identified in the ULF range for large (magnitude greater than 7) earthquakes such as Spitak, Loma Prieta, Guam, Biak etc. (see the review by Hayakawa and Hattori, 2004). That is, the ULF emissions are found to take place from a few weeks to a few days prior to large destructive earthquakes, which are considered as the so-called precursors of the general fracture. So that, we consider that these ULF emissions would carry the information on the microfracture taking place near the focal zone, or on the nonlinear dynamics of the lithosphere in association with an earthquake. This kind of nonlinear evolution can be effectively monitored by means of the fractal analysis. The first attempt with the use of fractal analysis was undertaken by Hayakawa et al. (1999) by using the slope in the frequency spectrum, and the similar analysis for the same Guam earthquake as in this paper has already been performed by Smirnova et al. (2001) and Gotoh et al. (2003, 2004), but in this paper we use the full waveform of the ULF data, unlike those by Gotoh et al. $(2003,2004)$ based on the filtered data, and also we pay attention to the much longerperiod prefracture behavior.

\section{ULF Data used and the 1993 Guam earthquake}

The details of the ULF data for the Guam earthquake have already been given in Hayakawa et al. (1999), but we have to repeat only the important points as follows. The Guan earthquake with magnitude $M s=8.2$, occurred on 8 August

Published by Copernicus GmbH on behalf of the European Geosciences Union and the American Geophysical Union. 


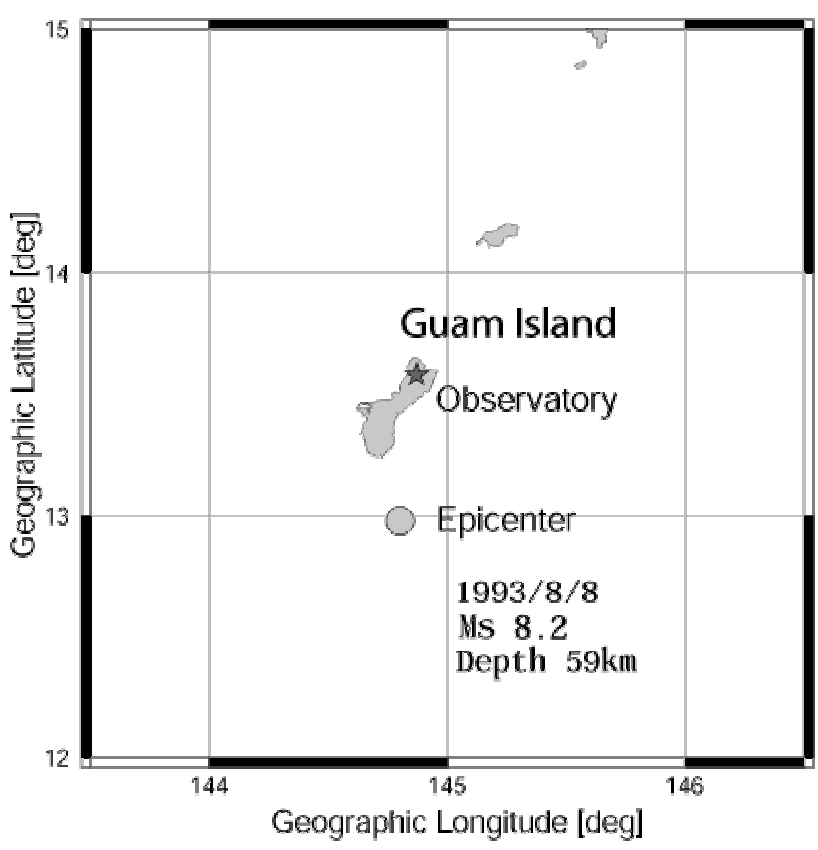

Fig. 1. Relative location of the ULF observing station (Guam) and the epicenter of 1993 August Guam earthquake.

1993 at 08:34 UT suddenly and without any foreshock activity. Its epicenter was located in the sea near the Guam island (geographic coordinates: $12.98^{\circ} \mathrm{N}, 1444.80^{\circ} \mathrm{E}$ ), and its depth was $60 \mathrm{~km}$. The Guam observatory where the ULF data were recorded, is located at $\sim 65 \mathrm{~km}$ from the epicenter. Figure 1 illustrates the relative location of our ULF observatory with respect to the epicenter. A regular magnetic observation is maintained there using a three-axis ring-coretype fluxgate magnetometer (Hayakawa et al., 1996). Three components of magnetic variations are usually recorded on a digital cassette tape with a sampling rate of $1 \mathrm{~s}$, but we use only the $H$ component (which is most intense). We analyze the data during one whole year of 1993, and we analyze the data during daytime ( $\mathrm{LT}=14: 00-15: 00)$, because Gotoh et al. (2004) have found that the most significant change in the monofractal dimension was observed for the Guam earthquake during daytime. One hour data are treated, so that the number of data is 3600 points per day.

\section{Fractal analysis by Higuchi method}

Different kinds of methods of fractal behavior of time series data have been proposed; (1) the spectral slope in frequency spectra (Hayakawa et al., 1999), (2) Burlaga and Klein (1986) method, (3) Higuchi (1988) method etc. Gotoh et al. (2003, 2004) have compared these methods extensively, and have suggested that the Higuchi method is superior to the methods (1) and (2) and that it is advised to use for the fractal analysis of the ULF data. This is found by Gotoh (2003) that the fractal dimension defined and used by Higuchi is most reasonable and acceptable in the sense of fractal definition. So that, we have followed their suggestion and we have used the Higuchi method for our following fractal analysis.

Here we explain briefly the Higuchi's method. The estimation of fractal dimension (D) of the time series data (in our case ULF data with sampling of $1 \mathrm{~s}$ ) is based on the estimation of the length of the curve $X(t)$. The fractal time series $X(t)$ of our concern is used to define the following new time series.

$\tilde{X}_{m}(k) ; X(m), X(m+k), X(m+2 k), \cdots$,
$X\left(m+\left[\frac{N-m}{k}\right] \cdot k\right)(m=1,2, \cdots, k)$

We estimate the curve length for each $\tilde{X}_{m}(k)$, and we call it $L_{m}(k)(m=1,2, \cdots, k)$.

$L_{m}(k)=\frac{\left\{\left[\frac{N-m}{k}\right]\right.}{\left.\sum_{i=1}^{k}|X(m+i k)-X(m+(i-1) \cdot k)| \frac{N-1}{\left[\frac{N-m}{k}\right] \cdot k}\right\}}$

where the term, $\frac{(N-1)}{\left[\frac{N-m}{k}\right] \cdot k}$ is the normalizing factor. Finally, the curve length is defined as the arithmetic average as follows.

$<L(k)>=\frac{\sum_{m=1}^{k} L_{m}(k)}{k}$

Then, we plot $<L(k)>$ versus $k(k=1,2, \cdots, 10)$ and we estimate the slope by fitting, leading to the estimation of $\mathrm{D}$.

\section{Analysis result and discussion}

We have to describe the essential differences of the present analysis from our previous studies (Gotoh et al., 2003, 2004) for the same 1993 Guam earthquake. Gotoh et al. (2003, 2004) have paid attention to the behavior just before the earthquake, so that this paper intends to study the nonlinear prefracture behavior over a sufficiently long time interval of the order of nearly one year. Secondly, our previous works (Gotoh et al., 2003, 2004) have been based on the filtered data, but we will utilize the complete waveform (1 s sampling) data in this paper. The daytime $(\mathrm{nT} 1=14-15 \mathrm{~h})$ data are used as in Gotoh et al. (2003).

Figure 2 is the analysis result over one whole year of 1993 , and the bottom panel illustrates the temporal evolution of the fractal dimension (D). We treat the daytime one-hour data, which means that we have one point for each day. This daily $\mathrm{D}$ is plotted by thin line, and the full line curve indicates the average value of $D$ running over \pm 5 days (as the total of 11 days). In order to indicate the statistical significance of the peaks in the fractal dimension $\mathrm{D}$, we have plotted the average 


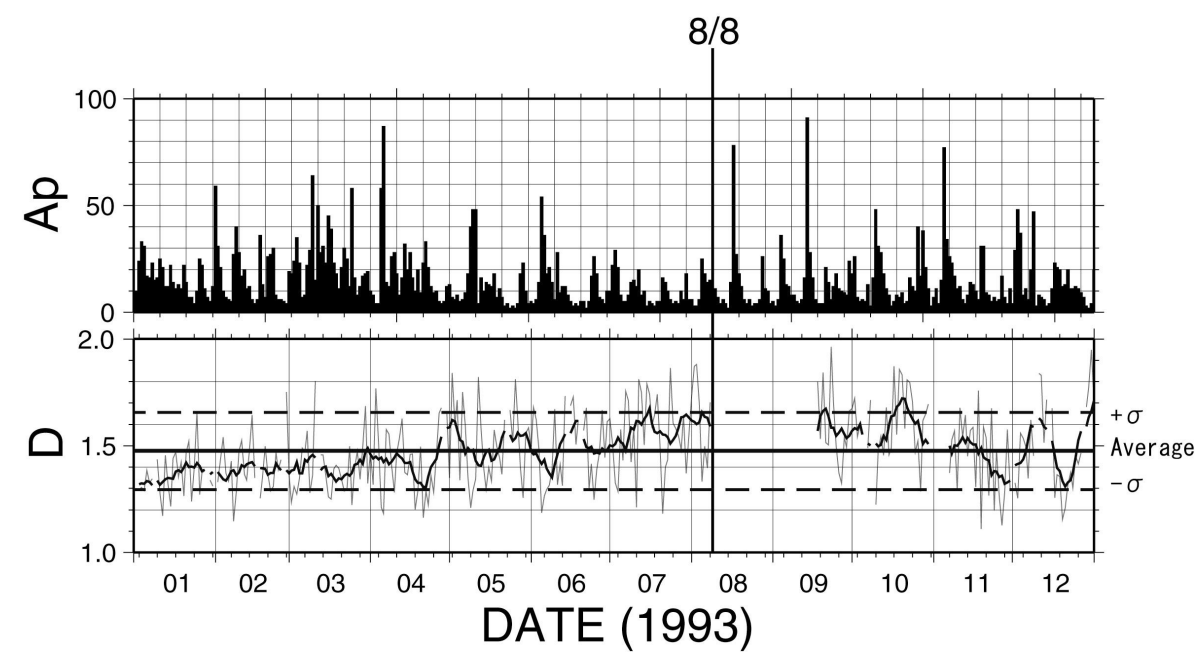

Fig. 2. Temporal evolution of fractal dimension (D) during one whole year of 1993 (bottom panel). The day of earthquake is indicated by a vertical line with the notation of $8 / 8$ (8 August). The upper panel indicates the geomagnetic activity expressed by Ap index. Daily D value is given in a thin line, the average value running over \pm 15 days is given by a thick line, the average $\mathrm{D}$ value over the whole period by a horizontal line and the $\pm \sigma$ ( $\sigma$ : standard deviation over the whole period) lines (by broken lines).

value for the whole period (as a horizontal line) and the $\pm \sigma$ ( $\sigma$ : standard deviation over the whole period) lines. We have found that average $\mathrm{D}=1.48$ and $\sigma=0.18$. The average $\mathrm{D}$ value is very close to that for the Brownian movement.

First of all, we look at the geomagnetic activity expressed by Ap index in Fig. 2. During three months before the earthquake, for example, starting in May, we understand from the figure that the geomagnetic activity is generally rather quiet. While, the geomagnetic activity is found to be significantly disturbed before May 1993. This means that the period of our concern before August 1993, is suitable for us to try to find any seismic effect in our ULF data because we can assume that solar-terrestrial effect is not so significant in this period.

Now we look at the temporal evolution of the fractal dimension (D) during one whole year of 1993. In the initial period of 1993, e.g., January, February and March, the value of $\mathrm{D}$ is found to remain at the background level of $\mathrm{D} \cong 1.4$. The running average (given in full line) of $\mathrm{D}$ gives us the approximate trend of $\mathrm{D}$, and then shows some kind of oscillations until the main shock, and the value of $\mathrm{D}$ increased up to $\mathrm{D}=1.8$. The dates of the peak values in $\mathrm{D}$ (in full line) are shown to be (1) 1 May, (2) 25 May, (3) 17 June, (4) 18 July and (5) two successive peak on 29 July and 4 August. When we look at the daily D value (in thin line) as the basis of the running average, the daily $\mathrm{D}$ value just around the peaks (or maxima) in the running average $\mathrm{D}$, is found to be very close to or to exceed the $2 \sigma$ line. This is indicative of the significance of those peaks in the running D. We now estimate the lead times of these significant peaks with respect to the main shock; (1) 99, (2) 75, (3) 52, (4) 21 and (5) 9 and 4 days before the main shock. A comparison of the time interval between the successive peaks suggests that the interval was about 20-30 days in the beginning of lithospheric change, but it becomes very short (of the order of 10-5 days) just before the earthquake main shock. After the main shock, there was a gap in the data due to the interruption of observation associated with the earthquake. In the middle of September, we have again the data and the fractal dimension (D) has shown the gradual depletion with some oscillations. Our fractal analysis is found to be very useful in finding out any nonlinear behavior in the lithosphere in possible association with the Guam earthquake. We have seen that the lithosphere have not exhibited a gradual change, but step-wise and discontinuous preparation toward the large Guam earthquake, and we have found that it took 3-4 months for a large earthquake to be prepared.

\section{Conclusions}

Though we have already found that the fractal dimension (by means of any analysis method) showed a significant change just before the Guam earthquake (Gotoh et al., 2003, 2004), this paper intends to study the longer-period nonlinear behavior of crustal activity before the 1993 Guam earthquake. Unlike the previous works (Gotoh et al., 2003, 2004) dealing with the filtered data, we have utilized the full waveform in this paper. We know that it takes more time for a large earthquake to be prepared, so that we want to know the longer-period (of the order of one year) property of the lithospheric activity than the previous works by Gotoh et al. $(2003,2004)$ before the 1993 Guam earthquake. Our fractal analysis based on Higuchi's method has elucidated the electromagnetic signature of prefracture criticality in such 
a way that the crustal movements must have taken place discontinuously (or step-wise) before the large earthquake. We could identify such discontinuous changes in the Earth's crust 99, 75, 52, 21 and 9, 4 days before the Guam earthquake.

Acknowledgements. The authors would like to express our sincere thanks to K. Yumoto of Kyushu University for initially providing us with the ULF data at Guam. One of the authors (M. Hayakawa) is grateful to Japan Society of Promotion of Science (\#15403012), the Mitsubishi Foundation and NiCT (R\&D promotion scheme funding international joint research) for their support.

Edited by: J. Kurths

Reviewed by D. Iudin and V. Lapenna

\section{References}

Burlaga, L. F. and Klein, L. W.: Fractal structure of the interplanetary magnetic field, J. Geophys. Res., 91, 347-350, 1986.

Gotoh, K., Hayakawa, M., and Smirnova, N.: Fractal analysis of the gromagnetic data obtained at Izu peninsula, Japan in relation to the nearby earthquake swarm of June-August 2000, Nat. Hazards Earth Syst. Sci., 3, 229-236, 2003, http://www.nat-hazards-earth-syst-sci.net/3/229/2003/.

Gotoh, K., Smirnova, N., and Hayakawa, M.: Fractal analysis of seismogenic ULF emissions, Special Issue on "Seismo Electromagnetics and Related Phenmena", edited by: Hayakawa, M., Molchanov, O. A., Biagi, B. F., and Vallianatos, V., Phys. Chem. Earth, Parts A/B/C, 29, 4-9, 419-424, 2004.

Hayakawa, M. and Fujinawa, Y. (Eds.): Electromagnetic Phenomena Related to Earthquake Prediction, Terra Sci. Pub. Co., Tokyo, 667p, 1994.
Hayakawa, M., Kawate, R., Molchanov, O. A., and Yumoto, K.: Results of ultra-low-frequency magnetic field measurements during the Guam earthquake of 8 August 1993, Geophys. Res. Lett., 23, 241-244, 1996.

Hayakawa, M., Itoh, T., and Smirnova, N.: Fractal analysis of ULF geomagnetic data associated with the Guam earthquake on $8 \mathrm{Au}-$ gust 1993, Geophys. Res. Lett., 26, 2797-2800, 1999.

Hayakawa, M. (Ed.): Atmospheric and Ionospheric Electromagnetic Phenomena Associated with Earthquakes, Terra Sci. Pub. Co., Tokyo, 996p, 1999.

Hayakawa, M. and Molchanov, O. A. (Eds.): Seismo Electromagnetics: Lithosphere-Atmosphere-Ionosphere coupling, TERRAPUB, Tokyo, 477p, 2002.

Hayakawa, M., Molchanov, O. A., and NASDA/UEC team: Summary report of NASDA's earthquake remote sensing frontier project, in: Special Issue on Seismo Electromagnetics and Related Phenomena, edited by: Hayakawa, M., Molchanov, O. A., Biagi, P. F., and Vallianatos, F., Phys. Chem. Earth, 29, 4-9, 617626, 2004.

Hayakawa, M. and Hattori, K.: Ultra-low-frequency electromagnetic emissions associated with earthquakes, IEEJ Trans. Fundamentals and Materials, 124, 12, 1101-1108, 2004.

Higuchi, T.: Approach to an irregular time on the basis of fractal theory, Physica D, 31, 277-283, 1988.

Molchanov O. A. and Hayakawa, M.: Generation of ULF seismogenic electromangetic emissions by microfracturing, Geophys. Res. Lett., 22, 3091-3094, 1995.

Molchanov, O. A. and Hayakawa, M.: On the generation mechanism of ULF seismogenic electromagnetic emissions, Phys. Earth Planet. Inter., 105, 201-210, 1998.

Smirnova, N., Hayakawa, M., Gotoh, K., and Volobuev, D.: Scaling characteristics of ULF geomagnetic field at the Guam seismoactive area and their dynamics in relation to the earthquake, Nat. Hazards Earth Syst. Sci., 1, 119-126, 2001, http://www.nat-hazards-earth-syst-sci.net/1/119/2001/. 